\title{
La matemática como herramienta para entender la economía dentro de la perspectiva de investigación de la Universidad Nacional Mayor de San Marcos ${ }^{1}$
}

\author{
Mathematics as a tool to understand the economy within the \\ research perspective of the Universidad Nacional Mayor de San \\ Marcos
}

Neisser Pino Romero ${ }^{2}$

\begin{abstract}
RESUMEN
La matemática desde sus inicios ha intentado describir la realidad en cual se vive, a través del tiempo ha ido apoyando a las diferentes ciencias las cuales mediante este soporte formal y abstracto ha sido enormemente fortalecido con el tiempo generando modelos matemáticos, formulas, expresiones que puedan resolver problemas reales en la medida que se pueda. Por mientras, que la economía donde se estudia y analiza los diferentes fenómenos que involucran la dinámica de generación de bienes y recursos, servicios y procesos en el mercado, por lo cual la economía ha necesitado de la matemática para formalizar de manera lógica con expresiones formales y abstractas pero con interpretaciones aplicadas a la realidad.
\end{abstract}

Palabras Clave: matemática; economía; modelamiento económico; formación universitaria; sistemas dinámicos.

1 Articulo con fines netamente académicos.

2 Docente de la Facultad de Ciencias Económicas de la Universidad Nacional Mayor de San Marcos. Docente de la Facultad de Ciencias y Filosofía "Alberto Cazorla Talleri" de la Universidad Peruana Cayetano Heredia. Investigador Visitante en Carleton University (Canada's Capital University). Licenciado en Computación Científica (UNMSM). Magíster en Matemática Aplicada con mención en Matemática Computacional (UNMSM). Email: neisser.pino@unmsm.edu.pe,neisser.pino@upch.pe

(C) Los autores. Este artículo es publicado por Pensamiento Crítico de la Facultad de Ciencias Económicas, Universidad Nacional Mayor de San Marcos. Este es un artículo de acceso abierto, distribuido bajo los términos de la licencia Creative Commons Atribucion - No Comercia_Compartir Igual 4.0 Internacional. (http:// creativecommons.org/licenses/by-nc-sa/4.0/) que permite el uso no comercial, distribución y reproducción en cualquier medio, siempre que la obra original sea debidamente citada. 


\begin{abstract}
Mathematics from its beginnings has tried to describe the reality in which one lives, over time has been supporting the different sciences of which with this formal and abstract support has been greatly strengthened over time generating mathematical models, formulas, expressions that Solve real problems as far as you can. For how much, that the economy where it is studied and analyzes the different phenomena that involve the dynamics of generation of goods and resources, services and processes in the market, reason why the economy has needed of the mathematics to formalize formally with formal expressions And abstract but with interpretations applied to reality.
\end{abstract}

Keywords: mathematics; economics; economic modeling; university education; dynamic systems.

Clasificación: JEL A22

\title{
1. Introducción
}

Desde los tiempos antiguos donde se realizaba el trueque, y después con algún objeto monetario, es donde la economía comienza a tomar una posición importante en la administración pecuniaria de cualquier persona, institución, sociedad, nación y más aún cuando se incrementó la relación entre diferentes países mediante la globalización se consideró a la matemática como la esencial y principal soporte para las decisiones que se puedan realizar ante problemas complicados. La estadística también ha aportado enormes herramientas para la recopilación, estudio, análisis y validación de datos que permitan considerar soluciones en corto y mediano plazo dentro del área de la economía que esté analizando el problema. La importancia de una ciencia se da en medida que pueda permitir obtener posibles respuestas ante los problemas con la mayor rigurosidad científica debido a que las soluciones que se puedan aplicar pueden tener implicancias de gran índole en la sociedad.

Según la opinión de Benegas (2016) en su artículo “Las matemáticas y la economía" deja un resumen de sus diversos comentarios, el cual sigue: "estos comentariossobrelas matemáticas se circunscriben a la imposibilidad de construir teorías económicas con base en ese instrumento que intenta medir lo inmedible, lo que, de más está decir, no invalida para nada su inmensa utilidad en otros muchos campos de investigación y en la misma vida diaria para evaluar proyectos." 
Realmente la matemática desde sus orígenes ha sido una manera de formalizar lo compleja que es la vida misma, este intento desde los egipcios hasta los griegos, luego con los avances de los romanos y la innovación de los árabes; inclusive desde los grandes matemáticos insignes de la matemática moderna como Leibniz o Newton comienzan a surgen nuevos horizontes para la matemática en vía de relacionarse con las demás ciencias, en primer lugar con la física luego con la biología y la economía, con respecto a la economía se tuvo grandes alcances de percepción de una ciencia social donde las ecuaciones matemáticas tenían la misión no solo de representar números y soluciones matemáticas sino más bien dar respuestas ante problemas suscitados en la sociedad en materia económica. Por tal, Gil (1988) dice lo siguiente: "la Economía es una ciencia esencialmente social que tiene como objeto la optimización de la asignación de los recursos escasos para la producción y distribución de bienes y servicios que satisfacen necesidades humanas."

Esta labor, claro está no es una tarea fácil de realizar en cuanto a los diversos problemas que se puedan suscitar y que cada problema tenga sus propias características y detalles a considerar si se desea resolver de una manera formal y ordenada que pueda representarse matemáticamente, y así ser resuelta mediante algún modelo matemático o alguna ecuación matemática.

Desde esta percepción se puede comentar o expresar diversos comentarios y apreciaciones sobre la importancia de la matemática en la economía y como la economía puede ser importante para la matemática aplicada.

El Grafico 1 representa el diagrama usual para la formulación de un modelo matemático que intenta resolver un problema real.

- Formular Dado un problema real, la primera labor es formular el problema con un lenguaje matemático. Las numerosas técnicas utilizadas en la construcción de modelos matemáticos van desde la consideración teórica del problema hasta la interpretación de los datos asociados con el problema. Por consiguiente, la mayoría de los modelos matemáticos implicaran funciones de una o más variables o ecuaciones que las definan (implícitamente). 
- Resolver Una vez construido el modelo matemático, se puede utilizar técnicas matemáticas apropiadas, las cuales permitirán resolver el problema.

- Interpretar Después de haber obtenido la solución matemática que se adecuada al modelo matemático, se tiene que interpretar los resultados en el contexto del problema real original.

- Probar Algunos modelos matemáticos de aplicaciones reales describen situaciones con una precisión completa. Pero otros modelos matemáticos dan, en lo mejor de los casos, una descripción aproximada del problema real. En este caso tener que probar la precisión del modelo observando qué tan bien describe el problema real y predice el comportamiento pasado o futuro. Si estos resultados no son satisfactorios, entonces puede que se tenga que volver a considerar los supuestos hechos en la construcción del modelo o, en el peor de los casos, iniciar de nuevo la construcción del modelo como se realiza en el paso Formular.

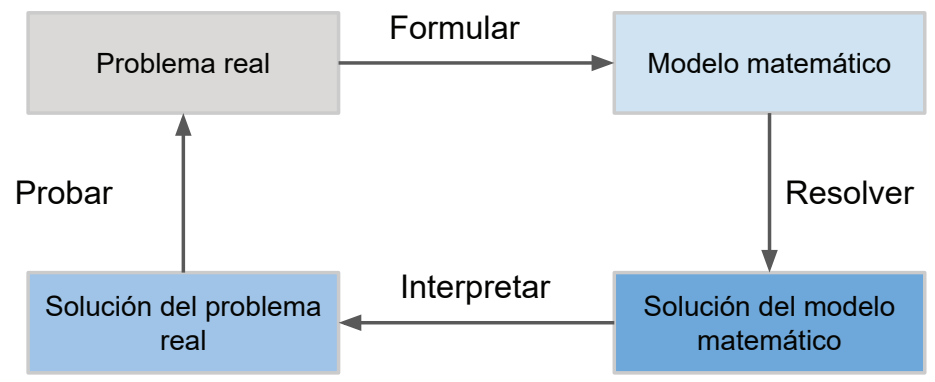

Grafico 1. Diagrama para la formulación de un modelo matemático

Fuente: Tan, Soon. Matemáticas Aplicadas a los Negocios, las Ciencias Sociales y la Vida.

Desde tiempo se ha considerado la Matemática como una ciencia complicada y abstracta para ser entendida por las otras ciencias por la poca aplicabilidad que se tiene, pero quizás sea el gran malentendido que se tiene en la comunidad científica para la formación de los estudiantes de la universidad. Es común escuchar de los estudiantes tantas expresiones 
que la matemática es difícil y/o un "curso no importante" el cual no debería ser impartido entre otras expresiones. Pero qué de cierto tiene estas afirmaciones en cuanto a la Matemática. Y cuáles podrían ser las respuestas adecuadas para una considerada formación matemática dentro de la Economía.

\section{Didáctica de la Matemática en la Economía}

La enseñanza de la matemática superior que se brinda en la universidad tiene un grado de exigencia mayor que la de la escuela en nivel secundario, pero que debe tener la adecuada base académica para poder seguir en el crecimiento de la abstracción de los problemas de la vida real en expresiones matemáticas. Y quizás el mayor problema que se puede percibir entre el nivel de la matemática se tiende a tener en cuenta que los problemas deben tener una solución “bonita” o de fácil acceso. Esta opinión hecha de mi parte, se debe que en mi etapa de estudiante universitario y de docente universitario siempre lo he notado cuando he impartido los cursos de matemática aplicada a la economía.

Se puede tener una adecuada enseñanza con una didáctica y una pedagogía idónea para que los estudiantes puedan aprender las diferentes técnicas de desarrollo, y quizás sea ese el detalle, el punto de quiebre que se tiene, que se enseña a utilizar las técnicas y nada más, sin considerar las demostraciones matemáticas de cómo se ha logrado obtener esas técnicas, es verdad que la economía utiliza las herramientas proporcionadas de la matemática pero hasta qué punto se utiliza la herramienta sin tener en cuenta la naturaleza del problema que se está resolviendo, o más bien hasta qué punto se presupone la existencia de la solución de un problema. Y una concepción que siempre se pueda conseguir una solución, cuando en muchos casos quizás no se pueda obtener la solución, o bajo qué circunstancias se pueda conseguir la solución del problema, lo cual permita obtener resultados, y por ende adecuadas interpretaciones sujetas a las restricciones que se han conseguido a través del análisis matemático. Son algunas de las referencias de los autores: Ortiz (2013), Moncada (2002) y Ruíz (1990) con respecto al desarrollo de la Matemática que ha relacionado temas de investigación en las diferentes ciencias, entre ellas la Economía. 
En la matemática, quizás el gran problema que tiene su desarrollo es la Existencia y la Unicidad de las Soluciones que debe demostrarse, y más aún la Positividad de las Soluciones para la adecuada resolución de un problema real que se intenta modelar matemáticamente. Muchos de los problemas que se presenta al resolver matemáticamente, solamente te indican hallar la solución; por lo cual se desarrolla el problema y se encuentra la solución y se interpreta la solución desde la perspectiva de la economía. Pero la interrogante sería ¿Qué sucede si no se encuentra la solución esperada? ¿Está mal planteado el problema propuesto?

\section{Modelamiento Matemático aplicado a la Economía}

Como se mencionaba en las secciones anteriores, el crear modelos matemáticos para ayudar a resolver problemas económicos tiene una misión y una visión a realizar en cuanto dar resultados acordes a la realidad y se pueda inferir adecuadas interpretaciones y la continuidad de su propio desarrollo para que pueda seguir brindando los resultados correctos dentro de las suposiciones que se tiene. Todo este proceso es sencillo de decir y de explicar de cómo se puede elaborar pero al momento de demostrar matemáticamente y validar el modelo con la realidad, he ahí el gran dilema que afronta el modelamiento económico.

En la presente sección comentaré de manera personal algunas experiencias sobre la matemática y la economía. Muchas de las experiencias pueden ser acertadas como equivocadas, como todo proceso en el desarrollo de la ciencia matemática como la económica. Tal vez, en este sentido sea necesario tener la perspectiva de una formación práctica sin muchos cuestionamientos de la problemática de la realidad. Y la economía debe dar respuestas en corto, mediano y largo plazo para lo cual necesita herramientas accesibles que le faciliten el problema en la medida que se pueda. La parte empírica de las situaciones y la recolección de datos que han sido de gran importancia en toda resolución de problemas mediante aproximaciones estadísticas permitieron una gran ayuda en brindar respuestas inmediatas y mediatas de acuerdo a la envergadura del problema y de las consecuencias de las decisiones que se puedan realizar. Muchos problemas son problemas en cadena que no solamente tienen que ver el aspecto económico sino más bien social que interactúan con diferentes variables que no son consideradas al momento de realizar un adecuado sistema que describa analíticamente el problema. 
Por su parte, Hersch (1999) define, con particular acierto, a la Matemática como "la Ciencia de objetos virtuales con propiedades reproducibles" -virtuales más como potenciales que como irreales-. Es decir, primero es una Ciencia, como las demás; pero sus objetos de estudio, y esto es lo específico de la Matemática, son ideales y virtuales. Así que es una Ciencia abstracta.

De la misma manera Fernández (2002) declara en su publicación "Lo imposible y la Ciencia abstracta", las Matemáticas son, desde luego, un lenguaje: un lenguaje diseñado para descubrir y describir patrones. ¿Y no es el objetivo de la Ciencia la búsqueda de patrones que permitan explicar fenómenos? Explicamos cuando encontramos simetrías, estructura, patrones que se repiten, que permanecen; y entendemos cuando somos capaces de organizar esas pautas en sencillos principios. Pero esos patrones son matemáticos y el lenguaje para analizarlos, combinarlos, para aplicarlos y obtener conclusiones, es el de las Matemáticas.

Por otro lado, según Salinas (2016) expresa lo siguiente: la economía se considera como ciencia porque utiliza el método científico para explicar las diversas teorías, modelos y sistemas económicos por medio de diversas leyes, mediante la investigación de campo o por medio de la observación de los hechos, que se aplican a las diversas teorías económicas. Y en otra expresión de su mismo trabajo manifiesta la referencia de la economía sobre su interacción con la sociedad: la economía es la ciencia que estudia la manera en que las sociedades deciden qué producir, cómo producirlo y para quién producirlo, tomando en cuenta que los recursos son escasos y las necesidades son ilimitadas. Y uno de los objetivos que consideramos que la ciencia económica se enfoca en el presente trabajo sería: descubrir las leyes del comportamiento económico de una sociedad y utilizar ese conocimiento para influir en el logro de objetivos sociales. Dado que la sociedad está constituida por distintos grupos e inevitablemente cada uno de ellos tendrá objetivos e intereses distintos que van cambiando con el tiempo, la economía adquiere un carácter político.

Teniendo las siguientes nociones que se tiene tanto de la Matemática como de la Economía, se podrá presentar un adecuado complemento para la formación universitaria de la Universidad Nacional Mayor de San Marcos. El modelamiento económico es una temática de gran importancia de 
analizar y de investigar debido que relaciona íntimamente la matemática y la economía. Y con una visión deductiva y constructiva se puede generar una concepción formal de los problemas económicos se puedan presentar en la sociedad. Esta consideración lo expresa el profesor Figueroa (2008) cuando manifiesta la dimensión social de la economía.

\subsection{Modelos matemáticos de una sola variable independiente}

Desde la formación académica de Matemática I, se empieza desde un sencillo recordatorio de la matemática antigua y medieval, para luego ingresar de manera abstracta de la matemática moderna, quizás en esta consideración el tema fundamental menos considerado como importante es la Teoría de Conjuntos, o de una manera más general denominado Topología. El motivo que empiezo con este tema como un tema ignorado es debido que en verdad es el tema fundamental y principal de donde todo tendrá sentido para un adecuado modelamiento matemático. Es decir, la existencia de las soluciones implica que exista en un adecuado conjunto (o espacio) con sus respectivas consideraciones. Por ejemplo: una persona está comprendida en el conjunto de los Números Enteros $(\mathbb{Z})$ y no en los números Reales $(\mathbb{R})$, si deseamos ser lo más precisos posibles además nos indicaría en donde están las soluciones.

A partir de los Conjuntos se comienza a construir los demás temas para poder crear modelos matemáticos, que comúnmente se expresan con funciones y en mayor medida con la derivada (razón de cambio instantáneo) donde se inicia el modelamiento matemático. De ahí se han ido generando las diferentes técnicas de optimización (maximización de utilidades y minimización de costos). Todas estas técnicas se van expresando mediante las funciones donde el tema principal que incluye la existencia de las funciones es el Dominio (como denominamos anteriormente en la teoría de los conjuntos). La continuidad de las funciones que significa que no tenga puntos no existentes en su dominio, luego más adelante se considerará el tema de los límites que será el punto de quiebre de toda investigación científica. De esta forma, la construcción de la matemática como un cimiento sólido para generación de conocimiento empieza para describir y contrastar la realidad con los modelos matemáticos que puedan dar respuestas a los problemas de la vida real. 
El motivo por el cual se considera que los límites son el punto de partida para la investigación científica donde la matemática comienza a ser la ciencia para formalizar los diferentes fenómenos de la realidad, es porque da la noción de superar la limitación de la no existencia en el denominador de una función racional.

- Idea clásica de la división

$$
\frac{a}{b}=k \quad \text { si } b \neq 0
$$

- Idea de la noción del limite

$$
\lim _{b \rightarrow 0} \frac{a}{b}=k^{*} \quad \text { cuando } b \neq 0
$$

El motivo del porque recordar estos temas tan conocidos y fundamentales de la matemática superior es debido que de ahora en adelante las limitaciones se intentan superar en la medida que se pueda. Los conocimientos se van desarrollando al intentar formalizar las ideas intuitivas que se puede inferir ante las dificultades de los problemas, y este proceso ha sido desde la antigüedad. La derivada y la integral que son definidos por el límite vienen a describir el inicio del modelamiento matemático en las diferentes áreas de las ciencias Biología, Química, Física y Economía. Del complemento de los operadores de la derivada y la antiderivada (integral) nacerán las Ecuaciones Diferenciales Ordinarias (E.D.O.) que describirán la mayoría de los modelos económicos, donde la teoría de Existencia y Unicidad de las Soluciones tomarán un gran fundamento en el análisis matemático debido que garantizar estos tópicos matemáticos formarán la base sólida de todo modelo matemático, más aún la positividad de las soluciones cuando se modela problemas de la realidad que no consideren cantidades negativas. Todas estas consideraciones solamente tendrán en cuenta a la variable independiente (exógena) del Tiempo. Pero ayudará enormemente a intentar formular los diferentes modelos y formalizar los pasos adecuados para una correcta construcción de un modelo matemático. 
El tema más delicado y más complicado de realizar un modelo matemático en cualquier área de la es la Estabilidad del Modelo (Local, Uniformemente, Asintóticamente, Global, etc.) debido que cuando uno realiza un sistema de ecuaciones, estos sistemas generalmente se dividen en dos tipos: los lineales y los no lineales. En la mayoría de los casos, las soluciones analíticas de los modelos lineales son sistemas de un análisis más sencillo de realizar con respecto a su estabilidad, y conocer que sucede a través del tiempo de manera directa, y por ende, obtener resultados e inferir interpretaciones adecuadas para el problema. Pero lamentablemente, la mayoría de los modelos matemáticos que intentan representar la realidad son del tipo No Lineal, por lo cual no es nada sencillo hallar las soluciones analíticas correspondiente del modelo. Ante este hecho común que un investigador, un estudiante interesado en aprender sobre los modelos matemáticos deben considerar otros medios para conseguir la estabilidad del modelo. Y las preguntas sobre diversos caminos de solución surgen en la mente del investigador.

¿Si puedo conseguir la solución analítica de un problema lineal, y no puedo conseguir, en la mayoría de los casos, de un problema No lineal; podría convertir un problema no lineal a un problema lineal?

Esta idea tan sencilla y curiosa dará inicio a un nuevo tipo de análisis en los modelos matemáticos que se le denomina análisis cualitativo de las soluciones, que estudiará el comportamiento de las soluciones en un largo plazo de tiempo. Ahora la perspectiva del estudio se enfocara sobre qué pasará en un largo periodo de tiempo, y más aún bajo qué perspectivas el modelo podría considerarse estable. Este análisis tiene un gran ámbito de estudio porque se centrará en todo un análisis delicado sobre las condiciones que puedan forzar la estabilidad. Más aun, el detalle fundamental de cómo la estabilidad de un modelo linealizado será la estabilidad del modelo no lineal original.

El problema original que es del tipo no lineal se ha asociado a un problema lineal mediante una aproximación polinomial: el polinomio de Taylor, por lo cual se halla las condiciones para determinar la estabilidad del modelo. Pero al realizar este proceso, surge otra complicación teórica, ¿la solución del problema linealizado es equivalente al problema no lineal original? Cada vez que se realiza un paso para lograr la estabilidad surge 
otra complicación, la investigación científica va logrando pasos notables, pero va encontrando más dificultades de las cuales surgen más técnicas de realizar un adecuado análisis de los modelos matemáticos. El crecimiento de la investigación matemática se va obteniendo debido a los diferentes problemas de gran impacto a través de la historia donde era necesario nuevos rumbos para lograr resultados correspondientes a las áreas de la ciencia que se están investigando.

Por otro lado, analizar cualitativamente el sistema lineal asociado requiere un conocimiento de todos los conceptos previos expuestos anteriormente, desde el espacio de las soluciones donde existan y sea única la solución para un problema de valor inicial, y también garantizar que las soluciones sean no negativas (soluciones positivas). A través de la historia, también se comenzó a generar un diferente tipo de análisis de los sistemas dinámicos en este caso las Ecuaciones Diferenciales Ordinarias que consistía en aproximar numéricamente las soluciones, este nuevo campo se denominó los Métodos Numéricos que comprendía diferentes técnicas dependiendo el tipo de sistema que se tuviera. Pero estas nuevas técnicas también tendrían sus detalles dentro de su área el cual consistía en el margen de error en el cálculo numérico. Desde aquí el modelamiento matemático obtuvo un adecuado complemento para realizar interpretaciones idóneas al problema que se está modelando. Pero desde un cálculo numérico surge las grandes cantidades de operaciones que se tiene que realizar por lo cual la ayuda computacional comienza a tomar una gran importancia debido a su rapidez y precisión dentro de su margen de error que pueda tener el computador, es decir, las computadoras también tienen limitaciones como la memoria RAM donde se reúne las operaciones y los cálculos donde para un procesamiento enorme de datos se debe realizar una optimización de algoritmos computacionales.

Realmente como todo en la ciencia matemática es un sendero de ideas intuitivas pero a la vez constructivas para ir generando nuevos senderos de investigación y de producción científica que ayudará a la ciencia, en particular a la economía, para el beneficio del desarrollo de la sociedad.

Hasta este punto, se ha considerado tres caminos fundamentales: el análisis matemático (soluciones analíticas), el análisis cualitativo (comportamiento de las soluciones) y el análisis numérico (soluciones 
aproximadas), donde el análisis cualitativo y el análisis numérico se complementarán cuando no se pueda realizar un análisis matemático directo y se pueda obtener las soluciones analíticas o exactas.

El modelamiento matemático en el sentido clásico se ha ido formando a través de los años mediante un trabajo interdisciplinario implícito donde la matemática ha ido relacionándose lentamente con las demás ciencias. Matemática con la economía, matemática con la física, matemática con biología, matemática con la química, especialmente con las ciencias básicas. Pero el modelamiento matemático no solo queda en los sistemas dinámicos que únicamente considere al tiempo como variable independiente sino que en los problemas sucede que hay más variables independientes por lo cual también deben formularse modelos matemáticos adecuados a estos tipos de problemas.

\subsection{Modelos matemáticos de varias variables independientes}

Después de haber realizado brevemente unos comentarios sobre la construcción de los modelos matemáticos y de qué manera va logrando una cierta complejidad en su creación y más que todo su validación tanto en su análisis matemático y la aplicabilidad al problema que está modelando. Estos conceptos van siendo la roca angular de todo lo que concierne el modelamiento matemático aplicado a la economía.

Uno de los grandes misterios más enormes que tiene la matemática que en la gran mayoría de casos, muchas de las técnicas que se realizan en un análisis matemático de una sola variable se puede extender a varias variables de una manera similar, una construcción similar pero con sus respectivos detalles y propiedades. La idea de generalización permite intuir que muchos de los resultados que se conocen se pueden deducir por lo cual la investigación tiene aires de seguir elevándose mucho más. Por lo cual, la situación de la investigación desde este momento donde los modelos de varias variables se considere algo complicado, tiene mucha razón debido que si se considera complicado en una sola variable, quizás con muchas variables independientes sea mucho más complicado. En esta parte se considera una construcción formal según expresado en el libro de "Real Analysis with Economic Applications" del professor Ok (2005), y el profesor Ortiz (2005) cuando menciona la historia de la Matemática a través de los años en las diferentes épocas del conocimiento. 
El modelamiento matemático empezó con las Ecuaciones Diferenciales Ordinarias (EDO) en un primer momento debido a la consideración de una sola variable independiente, pero con el paso del tiempo se fueron generando otros tipos de Ecuaciones Diferenciales donde también consideraba una sola variable independiente cuales las más resaltantes son las Ecuaciones Diferenciales con Retardo (EDR), las Ecuaciones Diferenciales Impulsivas (EDI), entre otras.

Cuando se tenía más variables independientes se comenzó a construir las Ecuaciones Diferenciales Parciales (EDP) donde despertaba un nuevo campo de análisis y de aplicaciones dinámicas en las ciencias básicas. Y desde ahí, la construcción deductiva de muchos de las técnicas que se realizaron en E.D.O. se comenzó a estructurar para las E.D.P. en la medida que fuera posible. Y de este estudio donde el mismo procedimiento es similar en la Existencia y Unicidad de las Soluciones, y más aún en la Positividad de las Soluciones, para luego realizar la estabilidad de los modelos matemáticos. Pero en relación a las E.D.P. se tenía una manera diferente en cuanto a la manera de la construcción delos modelos, y más aún el estudio analítico donde se diferenciara en dos tipos fundamentalmente: sentido clásico y sentido distribucional.

Desde momento, se considera dos rumbos diferentes en la investigación donde las ideas intuitivas que se generaron siempre para generar más investigación científica, donde ahora la premisa fundamental de estos modelos en E.D.P. se basa en las condiciones preliminares que se necesita para lograr los resultados esperados, si es que se puede demostrar satisfactoriamente los respectivos teoremas.

Y del mismo que se ha ido comentando como las ideas más intuitivas y con una gran perspectiva para ir logrando superar las limitaciones que siempre aparecen al realizar investigación matemática. De estas nociones viene la siguiente pregunta a realizarse.

¿Si para conseguir los resultados del modelo necesito cinco condiciones, podré conseguir resultados similares con tres condiciones únicamente, y más aún, si solamente tuviera una sola condición, podría obtener resultados similares al anterior de manera que las interpretaciones no varíen mucho? 
Ante estas interrogantes comienza ese nuevo sendero de lo que matemáticamente se le denomina debilitamiento de las soluciones, es decir, a menores condiciones requeridas para obtener resultados matemáticos se debilita la solución. Y de ahí, comúnmente se le denominará de la siguiente manera: soluciones fuertes (sentido clásico), y soluciones débiles (sentido distribucional).

A partir de estas soluciones se ha ido formando diferentes modelos matemáticos en las diversas áreas de la investigación de las ecuaciones diferenciales ya mencionadas, como las Ordinarias, las Parciales, las Estocásticas, las de Retardo, las Impulsivas, etc. Desde esta perspectiva la investigación y el desarrollo de un modelo matemático aplicado a la economía se sitúa en un estudio interdisciplinario entre la Economía y la Matemática por lo cual la formación de un estudiante de economía debe considerar en su propio desarrollado profesional. No hay duda que la formación matemática no es solo una herramienta de realización de operación sino más bien es una herramienta esencial para generar nuevos conocimientos necesarios para la economía en el día de hoy, donde dar respuesta ante problemas económicos de vital importancia para la sociedad en donde vivimos.

\section{Perspectiva de la Formación matemática aplicada a la Economía en la Universidad Nacional Mayor San Marcos (UNMSM)}

Desde muchos años atrás, se ha considerado la matemática como una adecuada herramienta en la cual se puede expresar muchas de las dinámicas, procesos, características de la realidad de donde se vive. La formación de los estudiantes en Economía bastaba en comprender el método tanto analítico y operativo para encontrar soluciones sin considerar los detalles más significativos de poder inferir la construcción del método y cómo se puede formar más resultados aperturando la investigación económica con un sólido fundamento matemático.

Lamatemática como una ciencia básica que contiene unaformalización y una abstracción tiene sus propios procesos y demostraciones para garantizar que los resultados matemáticos que brindan al servicio de las otras ciencias sean válidos, siempre y cuando las condiciones que requiere estos resultados se satisfagan adecuadamente. Esta ayuda enfocada de 
la mejor manera para construcción de expresiones económicas de tal manera que permitan describir los problemas de la realidad en cuanto sea posible expresarlas matemáticamente y así poder inferir interpretaciones económicas.

Las Escuelas Académicos Profesionales que están en la facultad de ciencias económicas de la universidad son tres: Economía, Economía Publica y Economía Internacional. Estas tres escuelas llevan cuatro cursos de matemática actualmente los cuales son: Matemática I, Matemática II, Matemática III y Matemática IV.

De estos cursos de Matemática, contienen los tópicos necesarios para poder generar modelos matemáticos desde su construcción, análisis, y validación. Estos cursos se relacionan con los cursos de Economía como microeconomía y macroeconomía especialmente.

El curso de Matemática I contiene los tópicos básicos del análisis matemático, es decir, la teoría sobre conjuntos, funciones, límites, continuidad, y derivadas. De este último tópico, se describe el proceso de optimización de funciones siendo la aplicación de las derivadas para la economía. Toda esta teoría están centradas en las funciones reales de variable real, es decir, estas funciones contienen una sola variable independiente. Por mientras, que el curso de Matemática II que contiene los temas de integral, álgebra lineal (espacios vectoriales, matrices, polinomio característico, autovalores y autovectores), luego sigue con el cálculo vectorial, es decir, todos los temas que se vieron en Matemática I pero con varias variables independientes. Con los temas básicos del análisis matemático se entra a la relación entre las derivadas e integrales, lo que son las Ecuaciones Diferenciales Ordinarias con todos sus tópicos respectivos (existencia y unicidad, resolución de las ecuaciones diferenciales lineales, convergencia de la solución) desarrolladas en Matemática III. Y para finalizar, el curso de Matemática IV, contiene el análisis cualitativo de las Ecuaciones Diferenciales Ordinarias, y una breve introducción a las Ecuaciones Diferenciales Parciales, no se tendrá en cuenta la optimización dinámica que contiene este curso, permitiendo tener la concepción de una variación (derivadas parciales) entre varias variables lo cual se puede generar modelos matemáticos aplicado a la economía. 
Un tópico que sirve para el modelamiento matemático que permite realizar simulaciones computacionales coherentes es el análisis numérico (soluciones numéricas o aproximadas) para lo cual se complementa adecuadamente con el análisis cualitativo de las Ecuaciones Diferenciales, lo que se ha expresado en la sección anterior. Este tópico apertura la concepción que se puede obtener aproximación de las soluciones que a veces no se puede obtener las soluciones analíticas, por lo cual se recurre al análisis del comportamiento de las soluciones. Estas simulaciones computacionales mediante los Métodos Numéricos permiten realizar aproximaciones numéricas de las soluciones donde estas soluciones pueden converger o divergen, numéricamente el software realiza las operaciones indicadas por el algoritmo computacional, pero la convergencia adecuada a la solución, lo va a determinar el análisis cualitativo para poder garantizar que la solución que se está aproximando sea la correcta. Por lo cual, las condiciones que garantizan la estabilidad mediante el análisis cualitativa, estas mismas condiciones permiten que la simulación computacional generada sea convergente, y más aún, esta solución numérica sea consecuente a la realidad del problema.

Según Wainer (2009), la perspectiva de realizar modelamiento computacional, que en muchos problemas de la realidad, suele ser como un casi imposible presentar mediante un sistema de ecuaciones el modelo matemático, por lo cual se tiene que buscar otras formas de resolver el problema y presentar respuestas, y soluciones adecuadas en tiempo real. Uno de estos métodos computacionales son los Autómatas Celulares (A.C.) que permite representar mediante reglas sencillas el proceso deinteracción de las variables para poder generar simulaciones computacionales en tiempo real para lograr obtener respuestas inmediatas. Desde aquí, en el modelamiento económico no solamente sería entre la matemática y la economía, sino también la computación para el procesamiento de datos y el modelamiento computacional. Las ciencias siempre se han podido relacionar de una manera u otra para generar conocimiento buscando que los fenómenos económicos puedan ser solucionados en la manera que sea posible teniendo en cuenta las consideraciones del problema y las limitaciones que se tiene en cualquier índole científica, es decir, la economía con las respuestas sociales que mediante un modelo matemático se intenta resolver, la matemática mediante las demostraciones y justificaciones de los resultados matemáticos para que sean válidos 
y aceptados como ciertos ante la comunidad científica, y por último, la computación con la limitación de procesar grandes cantidades de datos y operaciones necesarias para llegar a la solución deseada.

Por lo cual, de todo lo mencionado en la sección anterior, la intuición o mejor dicho la apertura a la curiosidad matemática de intentar romper las reglas clásicas falta considerable en la formación de los cursos matemáticos, lo cual no se expresa que sea sencillo de realizar pero si necesario para generar el interés en la investigación en la economía mediante los modelos matemáticos que son una fuente de problemas que permitirían crecer enormemente en la preparación analítica, critica de los problemas de realidad e intentarlos formalizarlos matemáticamente. La matemática no deja de sorprender a las mentes didácticas y curiosas que puede descubrir una estructura lógica-matemática para una construcción de estructuras matemáticas que puedan ayudar a la investigación científica en la economía. Finalmente, la formación matemática de una manera formal y demostrativa permitiría abrir la puerta hacia nuevos senderos de la investigación orientado hacia la economía, sin dejar de ser una herramienta para la economía para la descripción de los diversos problemas de la realidad en la que se vive.

\section{Conclusiones}

En verdad, surgen innumerables situaciones en que la economía y la matemática se pueden relacionar en la formación universitaria de un estudiante de economía. Pero también se presenta la disyuntiva en el área de la economía cada estudiante puede orientarse a especializarse, por lo cual, quizás el mayor rechazo hacia una matemática más constructiva y formal se pudiera ver retirada de la perspectiva de la investigación científica, como también puede ser el punto de partida para generar conocimientos de formación sólida en la economía mediante los modelos matemáticos aplicados a la economía, desde su construcción hasta su validación, desde su concepción de un problema real hasta la respuesta de la solución matemática. Todo la formación científica es la piedra angular de una adecuada preparación universitaria con miras hacia el futuro, en la sociedad que va cambiando a grandes pasos y cada vez más rápidos, donde la matemática aunque no se considere que permita encontrar soluciones por ser antigua, pero en realidad, la matemática sigue creciendo igualmente que la sociedad para poder describirla dentro de 
sus cimientos y perspectivas, teniendo en cuenta también las limitaciones que tiene también.

Por lo cual se puede tener las siguientes conclusiones de acuerdo a los libros que intentar relacionar de una manera complementaria entre la matemática y la economía en la investigación interdisciplinaria.

- La formación de un estudiante debe ser fundamentada en la construcción de modelos matemáticos aplicados a la economía de los problemas que afronta la sociedad en tiempo real.

- La matemática es una herramienta útil para poder formalizar problemas mediante modelos matemáticos aplicados a la economía que puedan dar respuestas a corto, mediano y largo plazo.

- La perspectiva de la enseñanza y la utilidad de la matemática para resolver problemas complejos permitiría generar la apertura a la investigación científica.

- La interdisciplinariedad entre la matemática y la economía como relación de investigación científica para la formación del estudiante.

- La apertura de romper límites en la investigación económica permitiría a la matemática crecer en sus métodos y aplicaciones, teniendo en cuenta las demostraciones matemáticas para garantizas los resultados matemáticos.

\section{Referencias Bibliográficas}

Benegas Lynch, Alberto. (2016). Las matemáticas y la economía. Instituto Mises Cuba. Punto de Vista Económico.

Fernández Gallardo, Pablo y Fernández Pérez, José Luis. (2002). Lo imposible y la Ciencia Abstracta. Universidad Autónoma de Madrid.

Figueroa, Adolfo. (2008). Nuestro Mundo Social: Introducción a la Ciencia Económica. Fondo Editorial PUCP. 292 páginas. 
Gil Ospina, Armando. (1998). La ciencia Matemática en la Economía. Revista Paginas. Número 53. Universidad Católica de Pereira. 10 páginas.

Hersch, R. (1999). What's Mathematics, really? Oxford University Pres

Moncada Araque, Keila L. (2002). Aplicación de la Matemática a la Economía. Dpto. de Pedagogía. Universidad de los Andes del Táchira. 232 páginas.

OK, Efe A. (2005). Real Analysis with Economic Applications. New York University. 600 páginas.

Ortiz Fernández, Alejandro. (2005). Historia de la Matemática, Volumen I. Sección Matemática. Pontificia Universidad Católica del Perú. 386 páginas.

Ortiz Fernández, Alejandro. (2013). Algunas Reflexiones sobre la EnseñanzaAprendizaje de la Matemática. Sección Matemática. Pontificia Universidad Católica del Perú. 350 páginas.

Ruiz Zúñiga, Ángel. (1990). Historia y Filosofía de las Matemáticas. Editorial UCR. Universidad de Costa Rica. 594 páginas.

Salinas, Cami. (2016). La economía como ciencia social: definición, métodos y objetivos. Universidad Autónoma de México.

Tan, Soon T. (2012). Matemáticas Aplicadas a los Negocios, las Ciencias Sociales y de la Vida. 920 páginas. Quinta Edición. Cengage Learning.

Wainer, Gabriel. (2009). Discrete Event Modelling and Simulation, A Practitioner's Approach. 483 páginas. CRC Press. 
NeISSER Pino RoMero 